\title{
TI.1.1
}

\section{Trust and Identity Document Stewardship}

- PDF: TI.1.1-TrustandldentityDocumentStewardship.pdf

- Text: TI.1.1-TrustandldentityDocumentStewardship.txt

\section{More Information}

\begin{tabular}{|l|l|}
\hline Repository ID & Tl.1.1 \\
\hline Persistent URL & http://doi.org/10.26869/TI.1.1 \\
\hline Title & Trust and Identity Document Stewardship \\
\hline Authors & David Walker, Tom Barton, Emily Eisbruch, Ann West, Mike Zawacki \\
\hline Sponsor & Internet2 \\
\hline Review & Document Stewardship Community Review \\
\hline Status & Preserve \\
\hline Publish Date & $4 / 18 / 2017$ \\
\hline DOI & $10.26869 /$ TI.1.1 \\
\hline Signature & \\
\hline Deprecated & No \\
\hline Future Review & $7 / 1 / 2017$ \\
\hline Supersedes & \\
\hline Format & \\
\hline Related Docs & \\
\hline Development Location & \\
\hline IP Framework & CC BY 4.0 \\
\hline Subject Tags & \\
\hline Notes & \\
\hline
\end{tabular}

